\title{
STABILITY INEQUALITIES INVOLVING GRAVITY NORM AND TEMPERATURE
}

\section{JiAJIN WEN, TIANYONG HAN AND JUN YUAN*}

Abstract. In the centered surround system $S^{(2)}\{P, \Gamma\}$, where $\Gamma$ is an ellipse and its eccentricity $\mathrm{e} \in[0,1)$, and $P$ is one of the foci of $\Gamma$, we establish the following stability inequalities:

$$
\sqrt{\frac{4 \pi}{15}} \times \frac{\mathrm{e}}{\sqrt{1-\mathrm{e}^{2}}} \leqslant \widetilde{\|\mathbf{F}\|} \leqslant \sqrt{2} \times \frac{\mathrm{e}}{\sqrt{1-\mathrm{e}^{2}}},
$$

where $\widetilde{\| \mathbf{F}} \|$ is the coefficient of variation of the gravity norm $\|\mathbf{F}\|$ and the coefficient $\sqrt{2}$ of $\mathrm{e} / \sqrt{1-\mathrm{e}^{2}}$ is the best constant. We also demonstrate the applications of the inequalities in the temperature change research, and obtain an approximate temperature coefficient of variation formula and an approximate temperature mean variance formula as follows:

$$
\widetilde{T} \approx 1.164752397618432 \cdots \times \frac{\mathrm{e}}{\sqrt{1-\mathrm{e}^{2}}}
$$

and

$$
\overline{\operatorname{Var}} T \approx 1.2897992775023233 \cdots \times \frac{\mathrm{e}}{\left(1-\mathrm{e}^{2}\right)^{3 / 2}} \times R_{\Gamma}^{-2} .
$$

Mathematics subject classification (2010): 26D15, 26E60, 51K05, 52A40, 62J10.

Keywords and phrases: Centered surround system, gravity norm, coefficient of variation, Newton formula, Euler formula.

\section{REFERENCES}

[1] A. Enciso, D. Poyato And J. Soler, Stability Results, Almost Global Generalized Beltrami Fields and Applications to Vortex Structures in the Euler Equations, Comm. Math. Phys. 360 (1) (2018), $197-$ 269.

[2] P. Zhao, Quantum variance of Maass-Hecke cusp forms, Comm. Math. Phys. 297 (2) (2010), 475 514.

[3] J. J. Wen, T. Y. HAN AND S. S. Cheng, Inequalities involving Dresher variance mean, J. Inequal. Appl., 2013: 366, 29 pages.

[4] J. J. WEn, Y. HuAng AND S. S. CHENG, Theory of $\phi$-Jensen variance and its applications in higher education, J. Inequal. Appl., 2015: 270 (2015), 40 pages.

[5] W. Mendenhall And T. Sincich, Statistics for Engineers and the Sciences (Fifth Edition), Upper Saddle River, New Jersey: Pearson Prentice Hall, 2006.

[6] C. F. PAGE, Allocation proportional to coefficients of variation when estimating the product of parameters, J. Amer. Statist. Assoc., 85 (412) (1990), 1134-1139.

[7] B. FleUry, O. GUÉDON AND G. PAOURIS, A stability result for mean width of Lp-centroid bodies, Adv. Math. 214 (2) (2007), 865-877.

[8] VAN DE MoORTEL, MAXIME, Stability and Instability of the Subextremal Reissner-Nordström Black Hole Interior for the Einstein-Maxwell-Klein-Gordon Equations in Spherical Symmetry, Comm. Math. Phys. 360 (1) (2018), 103-168.

[9] B. Ducomet AND E. FeIREISL, The equations of magnetohydrodynamics: on the interaction between matter and radiation in the evolution of gaseous stars, Comm. Math. Phys. 266 (3) (2006), 595-629. 
[10] C. D. Sijoy And S. Chaturvedi, Combining node-centered parallel radiation transport and higher-order multi-material cell-centered hydrodynamics methods in three-temperature radiation hydrodynamics code TRHD, Comput. Phys. Commun. 203 (2016), 94-109.

[11] R. Brunetti, K. Fredenhagen And K. RejZner, Quantum gravity from the point of view of locally covariant quantum field theory, Comm. Math. Phys. 345 (3) (2016), 741-779.

[12] J. J. WEN AND C. B. GAO, Geometric inequalities involving the central distance of the centered 2-surround system, Acta. Math. Sinica. 51 (4) (2008), 815-832. (in Chinese)

[13] C. B. GaO AND J. J. Wen, Theory of surround system and associated inequalities, Comput. Math. Appl. 63 (12) (2012), 1621-1640.

[14] C. B. GAO AND J. J. WEN, Theories and inequalities on the satellite system, ISRN Math. Anal., 2011, Article ID 909261, 22 pages.

[15] J. J. WEN, J. YAN AND S. H. WU, Isoperimetric inequalities in surround system and space science, J. Inequal. Appl., 2016: 74 (2016), 28 pages.

[16] J. J. WEN, J. YUAN, S. H. WU AND T. Y. HAN, Gravity inequalities and the mean temperature on a planet, J. Inequal. Appl., 2016: 264. 18 pages.

[17] J. J. WEN, S. H. WU, J. YUAN AND T. Y. HAN, Mean central distance-central distance inequalities, J. Math. Inequal., 11 (4) (2017), 1131-1149.

[18] J. J. Wen, T. Y. Han And C. B. GaO, Convergence tests on constant Dirichlet series, Comput. Math. Appl., 62 (9) (2011), 3472-3489.

[19] D. King-HeLE, Theory of satellite orbits in an atmosphere, Butterworths, London, 1964.

[20] C. B. GAO AND J. J. WEN, A dimensionality reduction principle on the optimization of function, J. Math. Inequal., 7 (3) (2013), 357-375.

[21] J. J. WEN, S. H. WU AND C. B. GAO, Sharp lower bounds involving circuit layout system, J. Inequal. Appl., 2013: 592 (2013), 22 pages.

[22] T. Y. HAN, S. H. WU AND J. J. WEN, Sharp upper bound involving circuit layout system, J. Nonlinear Sci. Appl. 9 (4) (2016), 1922-1935.

[23] F. R. K. ChUng AND PRAS AD TETALI, Isoperimetric inequalities for cartesian products of graphs, Combinatorics, Probability and Computing, 7 (2) (1998), 141-148.

[24] F. Della Pietra and N. Gavitone, The Relative isoperimetric inequality: the anisotropic case, J. Convex Anal., 20 (1) (2013), 157-180.

[25] J. J. WEN AND W. L. WANG, The inequalities involving generalized interpolation polynomial, Comput. Math. Appl., 56 (4) (2008), 1045-1058.

[26] J. J. Wen AND S. S. Cheng, Closed balls for interpolating quasi-polynomials, Comput. Appl. Math., 30 (3) (2011), 545-570.

[27] Z. H. YANG, Y. M. CHU AND W. ZHANG, Accurate approximations for the complete elliptic integral of the second kind, J. Math. Anal. Appl. 438 (2) (2016), 875-888.

[28] J. J. Wen, J. YUAN AND S. F. YUAN, An optimal version of an inequality involving the third symmetric means, Proc. Indian Acad. Sci. Math. Sci., 118 (4) (2008), 505-516.

[29] J. J. WEN AND W. L. WANG, The optimization for the inequalities of power means, J. Inequal. Appl., volume 2006, Article ID 46782, 25 pages.

[30] J. J. WEN AND W. L. WANG, Chebyshev type inequalities involving permanents and their applications, Linear Algebra Appl., 422 (1) (2007), 295-303.

[31] J. J. Wen, J. E. PeČARIĆ AND Y. H. TIAN, Weak monotonicity and Chebyshev type inequality, Math. Inequal. Appl., 18 (1) (2015), 217-231.

[32] J. J. Wen, C. B. GAO AND W. L. WANG, Inequalities of J-P-S-F type, J. Math. Inequal., 7 (2) (2013), 213-225.

[33] P. S. Bullen, P. S. Mitrinović And P. M. Vasić, Means and their inequalities, Reidel: Dordrecht/Boston/Lancaster/Toyyo, 1988. 